\title{
THE MOTION OF SUPERCONDUCTING VORTICES IN THIN FILMS OF VARYING THICKNESS*
}

\author{
S. J. CHAPMAN ${ }^{\dagger}$ AND D. R. HERON ${ }^{\dagger}$
}

\begin{abstract}
The interaction of superconducting vortices with superconductor/vacuum interfaces is considered. A vortex is first shown to intersect such an interface normally. Various thin-film models are then formulated, corresponding to different parameter regimes. A local analysis of a vortex is performed, and a law of motion for each vortex deduced. This law of motion implies that the vortex will move to the locally thinnest part of the film, and is consistent with the vortex moving under the curvature induced by being forced to intersect the boundaries of the film normally.
\end{abstract}

Key words. variable thickness, pinning

AMS subject classification. $82 \mathrm{D} 55$

PII. S0036139996307334

1. Introduction. There has been much recent interest in the consideration of a thin film of type II superconducting material in a perpendicular applied magnetic field, both from an experimental and a theoretical point of view $[1,17,18,19]$. Type II superconductors are characterized by the appearance of quantized, vortex-like structures of superconducting current. When a transport current is applied in the plane of such a film, vortices that are not pinned by impurities or other inhomogeneities in the material will move, with the direction of motion being perpendicular to the current. This motion of vortices causes energy to be dissipated, leading to an electrical resistance. It is therefore very important to understand the various mechanisms of pinning in this configuration. Here we study the effect of variations in thickness of the superconducting film on vortex motion and pinning.

Our starting point is the Ginzburg-Landau theory formulated in the static case by Ginzburg and Landau in 1950 [7], and in a time-dependent setting by Gor'kov and Éliashberg in 1968 [8]. The theory contains two important lengthscales, namely $\lambda$, the penetration depth, which represents the typical lengthscale for variations in the magnetic field, $\boldsymbol{H}$, and $\xi$, the coherence length, which represents the typical lengthscale for variations in the number density of superconducting electrons, which is effectively the vortex core radius.

The ratio of these two lengthscales is given by $\kappa=\lambda / \xi$, known as the GinzburgLandau parameter, and is often quite large.

Here we consider the limit in which $\kappa \rightarrow \infty$. The radius of the vortex cores then approaches zero when working on the $\lambda$ lengthscale, and the Ginzburg-Landau equations reduce to the simpler London equation

$$
\boldsymbol{\nabla}_{\wedge} \boldsymbol{\nabla}_{\wedge} \boldsymbol{H}+\boldsymbol{H}=2 \pi \delta(x) \delta(y) \boldsymbol{e}_{z},
$$

in which the delta function is due to a single vortex along the $z$ axis containing one quantum of flux, and $\boldsymbol{H}$ has been scaled with the flux quantum. Note that although in writing down (1.1) we have assumed that the Ohmic term $\sigma \partial \epsilon H / \partial t$ due to the normal

\footnotetext{
*Received by the editors July 26, 1996; accepted for publication (in revised form) February 11, 1997; published electronically August 3, 1998. The research of the first author was supported by a Royal Society Research Fellowship, and the research of the second author was supported by an EPSRC studentship.

http://www.siam.org/journals/siap/58-6/30733.html

${ }^{\dagger}$ Mathematical Institute, Oxford University, Oxford OX1 3LB, UK (chapman@maths.ox.ac.uk).
} 
electrons is small, there will still be dissipation as the vortices move around. This dissipation will come from three main sources: (i) in the vortex cores the Ohmic term is not small and there are normal currents flowing with their resulting dissipation, (ii) there will also be dissipation in the cores of the vortices due to the changing number density of superconducting electrons as the vortices move around, and (iii) even away from vortex cores an electric field is generated due to the changing magnetic field $\partial \epsilon H / \partial t$. Since this field will not in general be perpendicular to the current curl $\epsilon H$, there will be dissipation here also.

In section 2 we consider a straight vortex meeting a planar interface at an angle. The case of a perpendicular vortex was first considered by Pearl [14]. In this work and in [11] it is shown that the current near the surface of the superconductor decays only algebraically with distance, in contrast to the exponential decay in the bulk, with the conclusion that vortices interact much more strongly near the surface of the material. The magnetic field for a straight vortex at an angle to an interface has been considered by Kogan et al. [10]. Here we are interested in calculating the electric current in this situation, since it is the current that causes a vortex to move; we aim to show that the vortex must meet the boundary normally.

In section 3 we consider a system of vortices traversing a thin film of varying thickness. The problem of a single vortex traversing a film of uniform thickness was first considered by Pearl [13]. More recently Kogan [9] has derived equations for a system of vortices traversing a film of uniform thickness. Here we aim to generalize these models to films of varying thickness.

In section 4 we consider the effects on vortex motion of the variations in film thickness. We perform a local analysis of a single vortex and show that the vortex would travel to the locally thinnest point of the film. In section 5 we consider the effects of increasing the lateral dimensions of the film, and derive a new thin-film model. Finally we present some conclusions.

2. A straight vortex in a half-space. To begin our discussion of the interaction of vortices with boundaries, we consider a superconducting half-space $(z<0)$ containing a straight vortex, along the line $\Gamma$ given by $y=0, z=m x$, as shown in Figure 2.1.

The magnetic field $\boldsymbol{H}$ then satisfies

$$
\begin{array}{rlrl}
\boldsymbol{\nabla} \cdot \boldsymbol{H} & =0, & & \\
\boldsymbol{\nabla}_{\wedge} \boldsymbol{H} & =\mathbf{0} \quad \text { for } & z>0, \\
\boldsymbol{\nabla}_{\wedge} \boldsymbol{\nabla}_{\wedge} \boldsymbol{H}+\boldsymbol{H} & =2 \pi \boldsymbol{\delta}_{\Gamma} \quad \text { for } & & z<0,
\end{array}
$$

with

$$
\begin{aligned}
& {[\boldsymbol{H}]=\mathbf{0} \quad \text { on } \quad z=0 \text {, }} \\
& |\boldsymbol{H}| \rightarrow 0 \quad \text { as } \quad r \rightarrow \infty,
\end{aligned}
$$

where

$$
\boldsymbol{\delta}_{\Gamma}=\frac{\delta(y) \delta(x-z / m)}{m}\left(\begin{array}{c}
1 \\
0 \\
m
\end{array}\right)
$$

Equation (2.2) states that there is no current in the region external to the superconductor, while equation (2.3) is the London equation. The boundary condition (2.4) on 


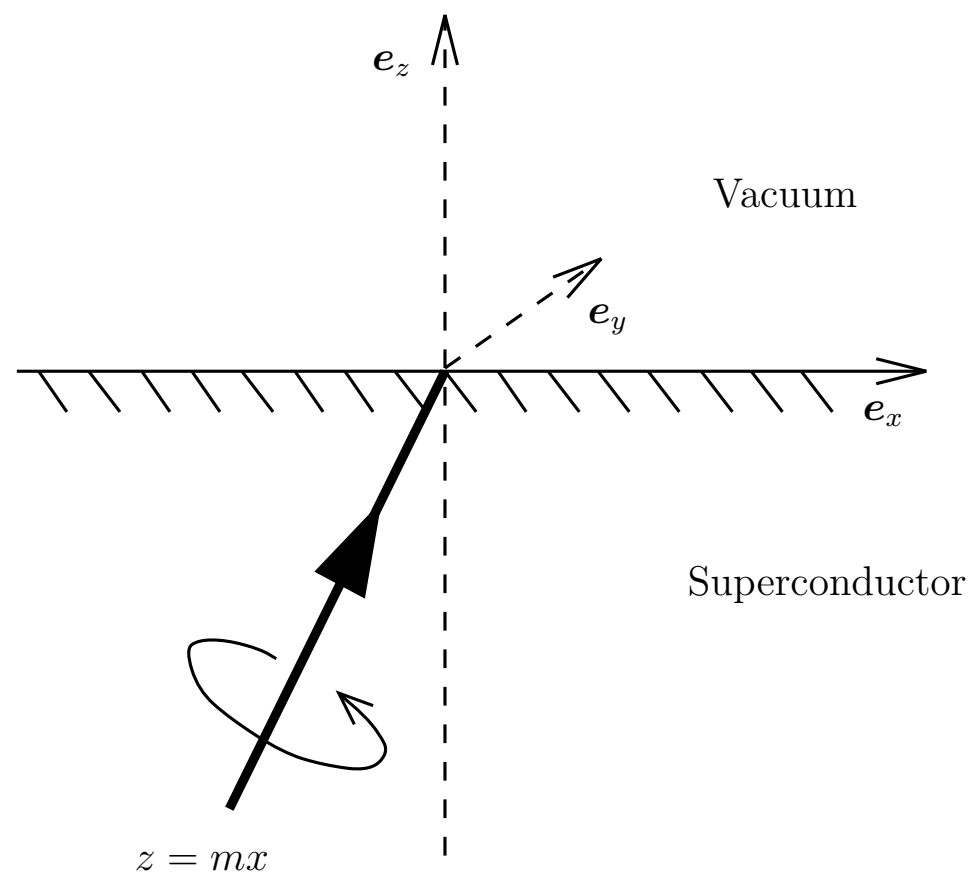

FIG. 2.1. An angled vortex in a half-space.

$z=0$ requires that $\boldsymbol{H}$ be continuous. We note that (2.2) and (2.4) imply $\boldsymbol{J} \cdot \boldsymbol{n}=0$ on $z=0$, where $\boldsymbol{J}=\operatorname{curl} \boldsymbol{H}$ is the electric current and $\boldsymbol{n}$ is the normal to the boundary.

Equations (2.1) and (2.2) imply that in $z>0$ we have $\boldsymbol{H}=\boldsymbol{\nabla} \phi, \nabla^{2} \phi=0$. Taking the double Fourier transform in $x$ and $y$ and requiring $\boldsymbol{H}$ to decay as $z \rightarrow \infty$ gives

$$
\widehat{\boldsymbol{H}}=\left(\begin{array}{c}
i k_{1} \\
i k_{2} \\
-k
\end{array}\right) \alpha\left(k_{1}, k_{2}\right) \mathrm{e}^{-z k} \quad \text { for } \quad z>0,
$$

where

$$
\widehat{\boldsymbol{H}}\left(k_{1}, k_{2}, z\right):=\int_{-\infty}^{\infty} \int_{-\infty}^{\infty} \mathrm{e}^{-i k_{1} x-i k_{2} y} \boldsymbol{H}(x, y, z) d x d y
$$

and

$$
k=\sqrt{k_{1}^{2}+k_{2}^{2}} .
$$

Transforming (2.1) and (2.3) gives

$$
\begin{aligned}
& i k_{1} \widehat{H}_{1}+i k_{2} \widehat{H}_{2}+\partial_{z} \widehat{H}_{3}=0 \\
& \partial_{z z}(\widehat{\boldsymbol{H}})-\left(k^{2}+1\right) \widehat{\boldsymbol{H}}=-\frac{2 \pi \mathrm{e}^{-i k_{1} z / m}}{m}\left(\begin{array}{c}
1 \\
0 \\
m
\end{array}\right) \quad \text { for } \quad \\
&
\end{aligned}
$$

Equation (2.7) gives

$$
\widehat{\boldsymbol{H}}=\boldsymbol{C} \mathrm{e}^{z\left(k^{2}+1\right)^{1 / 2}}+\frac{2 \pi m \mathrm{e}^{-i k_{1} z / m}}{k_{1}^{2}+m^{2}\left(k^{2}+1\right)}\left(\begin{array}{c}
1 \\
0 \\
m
\end{array}\right),
$$


where we have discarded the complementary function corresponding to exponential growth as $z \rightarrow-\infty$. The second term in (2.8) corresponds to the solution for a bulk vortex, since its inverse transform is given by

$$
\boldsymbol{H}=\frac{K_{0}\left(\sqrt{\frac{(m x-z)^{2}}{m^{2}+1}+y^{2}}\right)}{\sqrt{m^{2}+1}}\left(\begin{array}{c}
1 \\
0 \\
m
\end{array}\right),
$$

where $K_{0}$ is a modified Bessel function of the second kind. The first term in (2.8) contains the effects of the boundary. Substituting this form for $\widehat{\boldsymbol{H}}$ into equation (2.6) we obtain

$$
i k_{1} C_{1}+i k_{2} C_{2}+\left(k^{2}+1\right)^{1 / 2} C_{3}=0
$$

where $\boldsymbol{C}=\left(C_{1}, C_{2}, C_{3}\right)$. Continuity of $\boldsymbol{H}$ at $z=0$ gives the three conditions

$$
\left\{\begin{array}{l}
C_{1}=-P+i k_{1} \alpha \\
C_{2}=i k_{2} \alpha \\
C_{3}=-m P-k \alpha
\end{array}\right.
$$

where

$$
P=\frac{2 \pi m}{k_{1}^{2}+m^{2}\left(k^{2}+1\right)} .
$$

Eliminating the $C_{j}$ from equations (2.9) and (2.10) gives

$$
\alpha=-P \frac{\left(i k_{1}+m\left(k^{2}+1\right)^{1 / 2}\right)}{k\left(k+\left(k^{2}+1\right)^{1 / 2}\right)} .
$$

Thus we arrive at the solution for $\widehat{\boldsymbol{H}}$ given by

$$
\widehat{\boldsymbol{H}}=-P\left[\frac{\left(i k_{1}+m Q\right)}{k(k+Q)} \mathrm{e}^{-z k}\left(\begin{array}{c}
i k_{1} \\
i k_{2} \\
-k
\end{array}\right)\right] \quad \text { for } \quad z>0
$$

and

$$
\left\{\begin{array}{l}
\widehat{H}_{1}=-P\left[\left(1+\frac{i k_{1}\left(i k_{1}+m Q\right)}{k(k+Q)}\right) \mathrm{e}^{z Q}-\mathrm{e}^{-i k_{1} z / m}\right], \\
\widehat{H}_{2}=-P\left[\left(\frac{i k_{2}\left(i k_{1}+m Q\right)}{k(k+Q)}\right) \mathrm{e}^{z Q}\right], \\
\widehat{H}_{3}=-P\left[\left(m-\frac{\left(i k_{1}+m Q\right)}{(k+Q)}\right) \mathrm{e}^{z Q}-m \mathrm{e}^{-i k_{1} z / m}\right],
\end{array}\right.
$$

for $z<0$ where

$$
Q=\sqrt{k^{2}+1}
$$

We show in Figure 2.2 the field lines and the contours of $|\boldsymbol{H}|$ for the special case of the perpendicular vortex. 

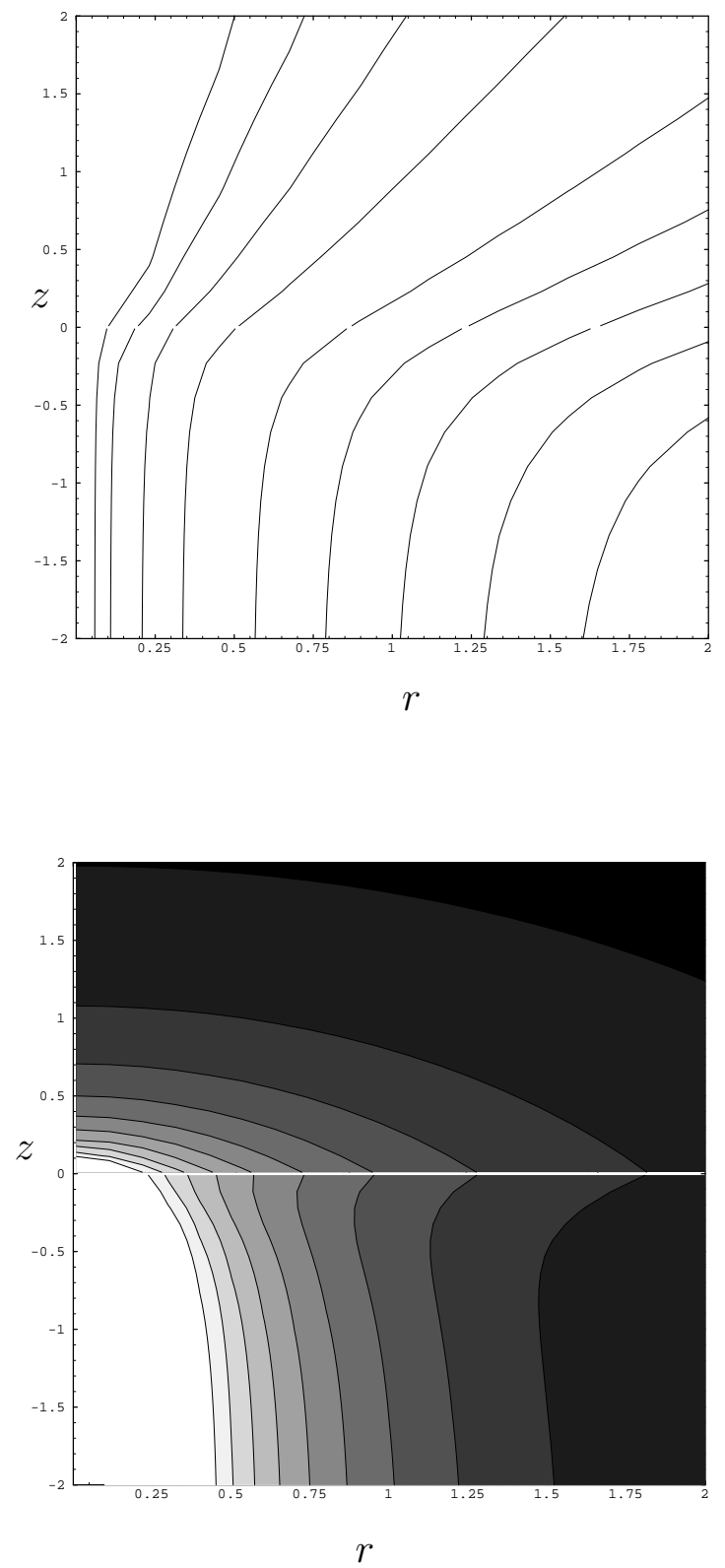

FIG. 2.2. The direction (top) and magnitude (bottom) of the magnetic field for the perpendicular vortex. In the bottom diagram the lighter regions indicate higher magnetic field magnitude and the contour values range from 0.1 to 1 in steps of 0.1 . 
We are primarily interested in the motion of the vortex, so we will focus now on the current, since it is the local current that causes the vortex to move. Because the vortex lies in the plane $y=0$, it is sufficient to consider the current in this plane only. It can be shown that the current on $y=0$ is given by $\boldsymbol{J}=J_{2} \boldsymbol{e}_{y}$, where $\boldsymbol{e}_{y}$ is the unit vector in the $y$ direction and

$$
\begin{aligned}
& J_{2}(x, 0, z)=-\frac{1}{4 \pi^{2}} \int_{-\infty}^{\infty} \int_{-\infty}^{\infty} \frac{P \mathrm{e}^{i k_{1} x+z Q}}{k}\left(i k_{1} m(Q-k)+k Q-k_{1}^{2}\right) d k_{1} d k_{2} \\
&-\frac{\left(m^{2}+1\right)}{4 \pi^{2} m} \int_{-\infty}^{\infty} \int_{-\infty}^{\infty} i k_{1} P \mathrm{e}^{i k_{1}(x-z / m)} d k_{1} d k_{2} \\
&= J_{\text {surf }}+ \\
& \text { bulk }
\end{aligned}
$$

Here the first term represents the current due to the surface interaction, while the second represents the bulk current due to the vortex. Indeed the bulk contribution can be integrated explicitly to give

$$
J_{\mathrm{bulk}}=\operatorname{sign}(x-z / m) \mathrm{K}_{1}\left(\frac{|m x-z|}{\sqrt{m^{2}+1}}\right)
$$

as expected.

The motion of a straight vortex is dictated by the nonsingular part of the current. The velocity of such a vortex can be derived from the Ginzburg-Landau theory [3], and is given by

$$
\boldsymbol{v}=\frac{2}{\beta}\left(\left.\left(\boldsymbol{J}-\boldsymbol{J}_{\text {bulk }}\right)\right|_{\text {at vortex }}\right) \wedge \boldsymbol{t},
$$

where $\boldsymbol{t}$ is the tangent vector to the vortex and $\beta$ is an order-one positive constant. Therefore, to consider the motion of the vortex, we need to analyze the surface interaction part of the current $\left(J_{\text {surf }}\right)$ at the vortex core. We proceed by using the change of variables $k_{1}=\rho \cos u, k_{2}=\rho \sin u$ on the first integral in equation (2.14), which becomes

$$
\begin{aligned}
J_{\text {surf }}= & -\frac{2 m}{\pi} \int_{\rho=0}^{\infty} \int_{u=0}^{\pi / 2} \frac{\left(\rho \sqrt{\rho^{2}+1}-\rho^{2} \cos ^{2} u\right) \mathrm{e}^{\rho z} \cos (x \rho \cos u)}{\left(m^{2}\left(\rho^{2}+1\right)+\rho^{2} \cos ^{2} u\right)} d u d \rho \\
& +\frac{2 m^{2}}{\pi} \int_{\rho=0}^{\infty} \int_{u=0}^{\pi / 2} \frac{\rho \cos u\left(\sqrt{\rho^{2}+1}-\rho\right) \mathrm{e}^{\rho z} \sin (x \rho \cos u)}{\left(m^{2}\left(\rho^{2}+1\right)+\rho^{2} \cos ^{2} u\right)} d u d \rho
\end{aligned}
$$

We set $z=m x$ and rescale $x=\epsilon X$ to look along the vortex, near the point of intersection. Now the integrals can be evaluated asymptotically by splitting the $\rho$ integration at $\epsilon^{-1 / 2}$. The second term in (2.18) is found to be order one in $\epsilon$, whereas the first can be integrated explicitly to leading order to give

$$
J_{\mathrm{surf}}=\frac{1}{2 \epsilon X m \sqrt{m^{2}+1}}+O\left(\epsilon^{-1 / 2}\right)
$$

Thus, using (2.16) unless $m$ is large, the vortex will move with infinite velocity at the tip in the $-\boldsymbol{e}_{x}$ or the $\boldsymbol{e}_{x}$ direction as $m$ is positive or negative. That is, the vortex will straighten to intersect normally with the boundary. 
Remark. We comment here on the analogy with fluid vortices, which are also found to intersect normally with rigid boundaries.

In the fluid case, the solution can be written down by introducing an equal and opposite image vortex, thus ensuring there is no component of the fluid velocity normal to the boundary. The vortex and its image form a vortex with discontinuous tangent at the point of intersection, and therefore infinite curvature. Since in the local induction approximation [16], the vortex velocity is proportional to its curvature, such a vortex would formally move with infinite speed (although, of course, the local induction approximation breaks down near parts of large curvature). Thus, the only stable configuration is that of a vortex meeting the boundary normally.

In the superconducting case, however, there is no simple image system, since the equations involve the magnetic field as well as the electric current, and must be solved both inside and outside the superconductor.

Locally near the point of intersection, however, the magnetic field decouples from the current, which then satisfies

$$
\begin{aligned}
& \boldsymbol{\nabla} \cdot \boldsymbol{J}=0, \\
& \boldsymbol{\nabla}_{\wedge} \boldsymbol{J}=\boldsymbol{\delta}_{\Gamma} \quad \text { for } \quad z<0, \\
& \boldsymbol{J} \cdot \boldsymbol{n}=0 \quad \text { on } \quad z=0,
\end{aligned}
$$

with solution

$$
\boldsymbol{J}=\boldsymbol{J}_{\text {vortex }}+\boldsymbol{J}_{\text {image }} .
$$

Now since the vortex and its image form a vortex with infinite curvature at the point of intersection, and since the velocity of a vortex is proportional to its curvature [3], the vortex moves with infinite speed. Note again though that this argument is purely formal, since the local induction approximation breaks down if the curvature becomes too large.

3. A thin film in a perpendicular field. In this section we consider applying a perpendicular magnetic field to a superconducting thin film of thickness order $d$, where $\xi \ll d \ll \lambda$. Hence the film will be thin on the lengthscale of the penetration depth but not as thin as a vortex core. We note that the case $d \ll \xi$ has been considered recently in $[5,2]$.

We wish to investigate how a slow variation in the thickness will affect the motion of the vortex. The variations we consider are, for simplicity, assumed to be symmetric about the $x y$ plane, but the results will be similar for arbitrary variations.

We begin by deriving a set of equations that describe several static vortices in a thin film of varying thickness. We then look at the dynamics of a single vortex and consider its motion due to these variations in thickness.

3.1. A thin film of symmetrically varying thickness. Suppose the typical lengthscale for thickness variations in the film is $\delta \lambda \gg \xi$ and the typical width of the film is $l \lambda$. We also define $a \lambda$ as the average separation of vortices; the size of $a$ is dictated by the applied field. Thus there are six lengthscales in the problem: $\xi, d, \delta \lambda$, $l \lambda, a \lambda$, and $\lambda$. We will consider the (in some sense canonical) case in which $a \sim \delta \sim l$, so that the variations in film thickness and the separation of vortices are comparable to the lateral dimension of the film.

For the rest of this section we will consider the case $l=1$, so that the film width is of the order of the penetration depth. Hence, in this section, we will continue 
to nondimensionalize all lengths with the penetration depth. We will consider the implications of larger film widths in section 5 . We therefore define the film to be given by $D=\left\{(x, y) \in \Omega \subseteq \mathcal{R}^{2},|z|<\epsilon g(x, y)\right\}$, where $g$ is order one, $\epsilon=d / \lambda$, and $x$, $y$ are nondimensional.

Our methodology involves an outer solution in which the film has zero thickness together with an inner solution within the film. We use capital letters to signify quantities in the film, lowercase for those external to it.

The equations to be satisfied are

$$
\begin{aligned}
\boldsymbol{\nabla}_{\wedge} \boldsymbol{J}+\boldsymbol{H} & =2 \pi \sum_{i} \delta\left(\boldsymbol{x}-\boldsymbol{x}_{i}\right) \boldsymbol{e}_{z} \quad \text { in } D \\
\boldsymbol{J} & =\boldsymbol{\nabla}_{\wedge} \boldsymbol{H} \quad \text { in } D, \\
\boldsymbol{\nabla} \cdot \boldsymbol{H} & =0 \quad \text { in } D, \\
\boldsymbol{\nabla}_{\wedge} \boldsymbol{h} & =\mathbf{0} \quad \text { outside } D, \\
\boldsymbol{\nabla} \cdot \boldsymbol{h} & =0 \quad \text { outside } D,
\end{aligned}
$$

with

$$
\begin{aligned}
\boldsymbol{h} & =\boldsymbol{H} \quad \text { on } \partial D, \\
\boldsymbol{J} \cdot \boldsymbol{n} & =0 \quad \text { on } \partial D,
\end{aligned}
$$

and

$$
\boldsymbol{h} \rightarrow h_{A} \boldsymbol{e}_{z} \quad \text { as } \quad|\boldsymbol{r}| \rightarrow \infty,
$$

where $\boldsymbol{n}$ is the normal to $\partial D$ and $\boldsymbol{x}=(x, y), \boldsymbol{r}=(x, y, z)$ are the two- and threedimensional position vectors.

We expand all quantities asymptotically in powers of $\epsilon$ :

$$
\begin{aligned}
\boldsymbol{h} & \simeq \boldsymbol{h}^{(0)}+\epsilon \boldsymbol{h}^{(1)}+\epsilon^{2} \boldsymbol{h}^{(2)}+\cdots, \\
h_{A} & \simeq h_{A}^{(0)}+\epsilon h_{A}^{(1)}+\epsilon^{2} h_{A}^{(2)}+\cdots, \\
\boldsymbol{H} & \simeq \boldsymbol{H}^{(0)}+\epsilon \boldsymbol{H}^{(1)}+\cdots, \\
\boldsymbol{J} & \simeq \boldsymbol{J}^{(0)}+\epsilon \boldsymbol{J}^{(1)}+\cdots
\end{aligned}
$$

We start by considering the problem inside the film. Rescaling $z=\epsilon Z$, equations (3.1) to (3.3) become

$$
\begin{aligned}
-\frac{1}{\epsilon} \frac{\partial J_{2}}{\partial Z}+\frac{\partial J_{3}}{\partial y}+H_{1} & =0 \\
\frac{1}{\epsilon} \frac{\partial J_{1}}{\partial Z}-\frac{\partial J_{3}}{\partial x}+H_{2} & =0 \\
\left(\frac{\partial J_{2}}{\partial x}-\frac{\partial J_{1}}{\partial y}\right)+H_{3} & =2 \pi \sum_{i} \delta\left(\hat{\boldsymbol{x}}-\hat{\boldsymbol{x}}_{i}\right) \\
\epsilon J_{1} & =-\frac{\partial H_{2}}{\partial Z}+\epsilon \frac{\partial H_{3}}{\partial y} \\
\epsilon J_{2} & =\frac{\partial H_{1}}{\partial Z}-\epsilon \frac{\partial H_{3}}{\partial x}
\end{aligned}
$$




$$
\begin{aligned}
J_{3} & =\left(\frac{\partial H_{2}}{\partial x}-\frac{\partial H_{1}}{\partial y}\right), \\
\frac{\partial H_{1}}{\partial x}+\frac{\partial H_{2}}{\partial y}+\frac{1}{\epsilon} \frac{\partial H_{3}}{\partial Z} & =0 .
\end{aligned}
$$

Substituting the expansions (3.9)-(3.12) into (3.13)-(3.19) and equating powers of $\epsilon$ gives, at leading order,

$$
\begin{aligned}
& \frac{\partial J_{1}^{(0)}}{\partial Z}=\frac{\partial J_{2}^{(0)}}{\partial Z}=0, \\
& \frac{\partial H_{1}^{(0)}}{\partial Z}=\frac{\partial H_{2}^{(0)}}{\partial Z}=0, \\
& \frac{\partial H_{3}^{(0)}}{\partial Z}=0 .
\end{aligned}
$$

Continuity in the field on the upper and lower faces of the film implies

$$
\left.\boldsymbol{h}\right|_{z= \pm \epsilon g}=\left.\boldsymbol{H}\right|_{Z= \pm g} \quad \text { for } \quad x, y \in \Omega .
$$

Expanding (3.23) in $\epsilon$ gives, at leading order,

$$
\left.\boldsymbol{h}^{(0)}\right|_{z=0^{ \pm}}=\left.\boldsymbol{H}^{(0)}\right|_{Z= \pm g} \quad \text { for } \quad x, y \in \Omega .
$$

Equations (3.21) and (3.22) imply that $\boldsymbol{H}^{(0)}$ is constant in $Z$, and so the problem for the leading-order external magnetic field, $\boldsymbol{h}^{(0)}$, becomes

$$
\begin{aligned}
\nabla_{\wedge} \boldsymbol{h}^{(0)} & =\mathbf{0}, \\
\boldsymbol{\nabla} \cdot \boldsymbol{h}^{(0)} & =0,
\end{aligned}
$$

with

$$
\begin{aligned}
{\left[\boldsymbol{h}^{(0)}\right] } & =\mathbf{0}, \\
\boldsymbol{h}^{(0)} & \rightarrow h_{A}^{(0)} \boldsymbol{e}_{z} \quad \text { as } \quad|\boldsymbol{r}| \rightarrow \infty,
\end{aligned}
$$

where the square brackets indicate the jump in the quantity enclosed across $\Omega$. Thus the solution is simply that the order-one applied magnetic field passes straight through the film such that

$$
\boldsymbol{h}^{(0)}=\boldsymbol{H}^{(0)}=h_{A}^{(0)} \boldsymbol{e}_{z},
$$

with $J_{1}^{(0)}, J_{2}^{(0)}$ constant in $Z$.

We return to the problem inside the superconductor. Equating powers of $\epsilon$ at the next order in (3.13)-(3.19) gives

$$
\begin{aligned}
\frac{\partial J_{1}^{(1)}}{\partial Z} & =\frac{\partial J_{2}^{(1)}}{\partial Z}=0, \\
\frac{\partial J_{2}^{(0)}}{\partial x}-\frac{\partial J_{1}^{(0)}}{\partial y}+h_{A}^{(0)} & =2 \pi \sum_{i} \delta\left(\boldsymbol{x}-\boldsymbol{x}_{i}\right), \\
J_{1}^{(0)} & =-\frac{\partial H_{2}^{(1)}}{\partial Z},
\end{aligned}
$$




$$
\begin{aligned}
J_{2}^{(0)} & =\frac{\partial H_{1}^{(1)}}{\partial Z}, \\
J_{3}^{(0)} & =0 \\
\frac{\partial H_{3}^{(1)}}{\partial Z} & =0 .
\end{aligned}
$$

Since $\boldsymbol{J}^{(0)}$ is constant in $Z,(3.32)$ and (3.33) can be integrated to give

$$
\begin{aligned}
& H_{1}^{(1)}=Z J_{2}^{(0)}, \\
& H_{2}^{(1)}=-Z J_{1}^{(0)} .
\end{aligned}
$$

Equating coefficients of $\epsilon$ in the expansion of (3.23) gives

$$
\left.\boldsymbol{h}^{(1)}\right|_{z=0^{ \pm}}=\left.\boldsymbol{H}^{(1)}\right|_{Z= \pm g} \quad \text { for } \quad x, y \in \Omega
$$

since $\frac{\partial \boldsymbol{h}^{(0)}}{\partial z}=\mathbf{0}$.

We are now in a position to state the problem for the first-order external field $\boldsymbol{h}^{(1)}$ in terms of the current $\boldsymbol{J}^{(0)}$. We have

$$
\begin{aligned}
\nabla_{\wedge} \boldsymbol{h}^{(1)} & =\mathbf{0}, \\
\boldsymbol{\nabla} \cdot \boldsymbol{h}^{(1)} & =0,
\end{aligned}
$$

with

$$
\begin{aligned}
{\left[\boldsymbol{e}_{z} \wedge \boldsymbol{h}^{(1)}\right] } & =2 g \boldsymbol{J}^{(0)} \quad \text { for } \quad(x, y) \in \Omega, \\
\boldsymbol{h}^{(1)} & \rightarrow h_{A}^{(1)} \boldsymbol{e}_{z} \quad \text { as } \quad|\boldsymbol{r}| \rightarrow \infty
\end{aligned}
$$

We note that the magnetic field generated by an order-one current in the film is $O(\epsilon)$.

Thus, given $\boldsymbol{J}^{(0)}$, we can calculate $\boldsymbol{h}^{(1)}$. To complete the problem for $\boldsymbol{J}^{(0)}$ we consider the third component of equation (3.39) evaluated on the upper surface of the film, namely

$$
\frac{\partial h_{1}^{(1)}}{\partial y}(x, y, \epsilon g)=\frac{\partial h_{2}^{(1)}}{\partial x}(x, y, \epsilon g) .
$$

Expanding in $\epsilon$ and using equations (3.36) to (3.38) gives

$$
\boldsymbol{\nabla} \cdot\left(g \boldsymbol{J}^{(0)}\right)=0 .
$$

We can rewrite (3.31) in the vector form

$$
\boldsymbol{e}_{z} \cdot \nabla_{\wedge} \boldsymbol{J}^{(0)}+h_{A}^{(0)}=2 \pi \sum_{i} \delta\left(\boldsymbol{x}-\boldsymbol{x}_{i}\right) .
$$

We note that a similar equation to this has been previously derived in [9] for the case of constant thickness, by averaging across the film. To complete this decoupled problem for the leading-order current we include the leading-order behavior of (3.7), namely

$$
\boldsymbol{J}^{(0)} \cdot \boldsymbol{\nu}=0 \quad \text { on } \quad \partial \Omega,
$$


where $\boldsymbol{\nu}$ is the unit outward normal to $\partial \Omega$.

We note that there are two source terms in equation (3.45) for the current. The first is the sum of $\delta$-functions due to the vortices, as expected. The second is due to the applied magnetic field, which acts as a negative distributed vorticity. This term is due to the Meissner effect, by which a superconductor attempts to exclude a magnetic field from its interior. The current generated by this term in (3.45) is an attempt to shield the superconductor from the applied field, and will result in a lower magnetic field inside the superconductor than the applied field $h_{A}^{(0)}$.

In the next section we study the problem (3.44)-(3.46) for the leading-order current in the case of a single vortex and deduce an equation for the velocity of a vortex in a thin film of varying thickness.

4. A near core analysis of a single vortex in a thin film of varying thickness. Here we aim to investigate the effects of varying film thickness on vortex motion by looking locally near a single vortex using matched asymptotic expansions. The method was first introduced by Neu [12] for complex scalar fields, and has since been employed by a variety of authors for various situations in bulk superconductors $[3,4,6,15]$.

Decomposing $\boldsymbol{J}^{(0)}$ into $\boldsymbol{\nabla}_{\wedge}\left(F \boldsymbol{e}_{z}\right)+\boldsymbol{\nabla} p$, with $F=F(x, y)$ and $p=p(x, y)$, and considering a single vortex at the origin, we can rewrite equations $(3.44),(3.45)$ as

$$
\begin{aligned}
F & =-\log r+h_{A}^{(0)} \frac{r^{2}}{4}, \\
\nabla \cdot(g \nabla p) & =-\left(\nabla_{\wedge}\left(F \boldsymbol{e}_{z}\right)\right) \cdot \boldsymbol{\nabla} g,
\end{aligned}
$$

which implies

$$
\boldsymbol{J}^{(0)}=\left(\frac{1}{r}-h_{A}^{(0)} \frac{r}{2}\right) \boldsymbol{e}_{\theta}+\nabla p
$$

where $r=\sqrt{x^{2}+y^{2}}$. We note that the current due to the vortex decays only algebraically with distance, in contrast to the exponential decay for a vortex in a bulk material, so that the interaction between vortices should be stronger in thin films, as noted by [13].

To investigate the effects of the varying thickness on the motion of the vortex we examine the solution of (4.2) as the vortex is approached. We assume that the model is quasi-static, that is, that the motion of the vortex is slow enough that the magnetic field relaxes instantly. This corresponds to the relaxation time for the order parameter being much greater than that for the magnetic field in the time-dependent Ginzburg-Landau equations.

We assume, and it can be justified a posteriori, that

$$
g \nabla^{2} p \gg \nabla g \cdot \nabla p \text { as } r \rightarrow 0 .
$$

Then

$$
\nabla^{2} p \sim-\frac{\boldsymbol{e}_{\theta}}{r g(0,0)} \cdot\left(g_{x}(0,0), g_{y}(0,0)\right) \text { as } r \rightarrow 0,
$$

and therefore

$$
p \sim \frac{1}{2 g_{0}}\left(\log r\left(y g_{x 0}-x g_{y 0}\right)+C_{1} x+C_{2} y\right) \text { as } r \rightarrow 0,
$$


where $C_{1}, C_{2}$ are constants and $g_{0}=g(0,0), g_{x 0}=g_{x}(0,0), g_{y 0}=g_{y}(0,0)$. Hence

$$
\nabla p \sim \frac{1}{2 g_{0}}\left(\begin{array}{c}
-\left(\log r+\frac{x^{2}}{r^{2}}\right) g_{y 0}+\frac{x y}{r^{2}} g_{x 0}+C_{1} \\
\left(\log r+\frac{y^{2}}{r^{2}}\right) g_{x 0}-\frac{x y}{r^{2}} g_{y 0}+C_{2}
\end{array}\right) \text { as } r \rightarrow 0,
$$

so that (4.4) holds. Hence the current satisfies

$$
\boldsymbol{J}^{(0)} \sim \frac{1}{r} \boldsymbol{e}_{\theta}+\frac{1}{2 g_{0}}\left(\begin{array}{c}
-\left(\log r+\frac{x^{2}}{r^{2}}\right) g_{y 0}+\frac{x y}{r^{2}} g_{x 0}+C_{1} \\
\left(\log r+\frac{y^{2}}{r^{2}}\right) g_{x 0}-\frac{x y}{r^{2}} g_{y 0}+C_{2}
\end{array}\right) \text { as } r \rightarrow 0 .
$$

As in the case of the bulk vortex, to determine the vortex motion we need to analyze the core of the vortex using the full Ginzburg-Landau theory. We rescale $x=X / \kappa, y=Y / \kappa$, giving

$$
\boldsymbol{J} \sim \frac{\kappa}{R} \boldsymbol{e}_{\theta}+\frac{\log \kappa}{2 g_{0}}\left(\begin{array}{c}
g_{y 0} \\
-g_{x 0}
\end{array}\right)+\cdots .
$$

We hope to match this expression with a solution to the Ginzburg-Landau equations in the core region. Fortunately, the current surrounding a curvilinear vortex as the vortex is approached has already been derived in [3]. Following that work, we let the vortex lie along a curve $\Gamma$, given by $\boldsymbol{r}=\boldsymbol{q}(s)$, and define a local coordinate system by

$$
\boldsymbol{r}=\boldsymbol{q}(s)+\frac{\tilde{X}}{\kappa} \boldsymbol{n}(s)+\frac{\tilde{Y}}{\kappa} \boldsymbol{b}(s),
$$

where $s$ is arclength, and $\boldsymbol{n}(s), \boldsymbol{b}(s), \boldsymbol{t}(s)$ are the unit normal, binormal, and tangent, respectively, to the curve $\Gamma$. The expression for the current that matches the Ginzburg-Landau solution for a curvilinear vortex is then given in [3] as

$$
\boldsymbol{J} \sim \frac{\tilde{\boldsymbol{e}}_{\theta}}{\tilde{R}}\left\{\kappa+\frac{K \tilde{X}}{2}\right\}+\frac{K \boldsymbol{b}}{2}\left\{-\log \left(\frac{\tilde{R}}{\kappa}\right)+\log 2-1\right\}+\cdots,
$$

where $\tilde{R}$ and $\tilde{\theta}$ are local polar coordinates and $K$ is the curvature of the vortex. Matching with the inner solution near the vortex core gives the local vortex velocity as

$$
\boldsymbol{v}=\frac{K}{\beta}(\log \kappa) \boldsymbol{n}+O(1)
$$

We need to relate the curvilinear local coordinates $\tilde{X}, \tilde{Y}$ to the Cartesian coordinates $X, Y$.

Assuming that the vortex is a planar curve, we define $\gamma$ as the acute angle between the principal normal to $\Gamma$ at $\boldsymbol{r}=\boldsymbol{q}(s)$ and the line AO in Figure 4.1, and $\phi$ as the acute angle between the line $\mathrm{AO}$ and the $\boldsymbol{e}_{x}$ axis. We have already seen that $\Gamma$ must meet the surfaces of the film normally, and we find that it is approximately vertical to leading order, so that

$$
\cos \gamma \sim 1, \quad \sin \gamma=O(\epsilon)
$$




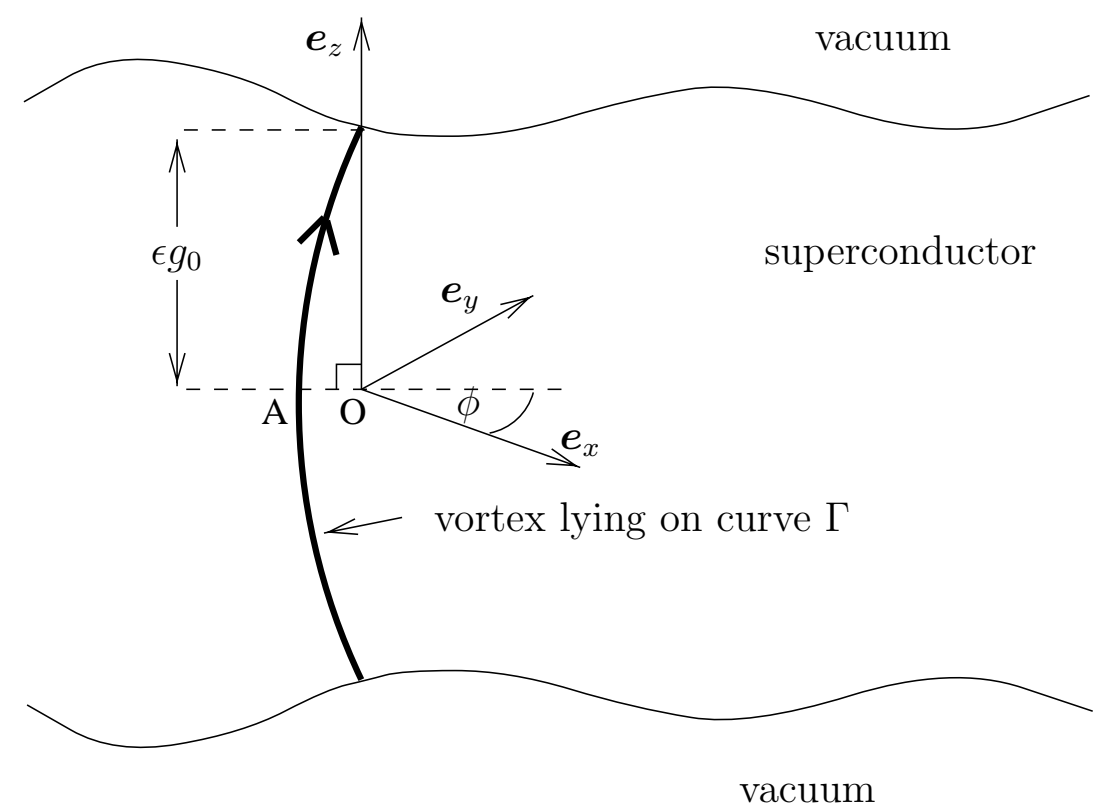

FIG. 4.1. The geometry of a vortex in a thin film of varying thickness.

and

$$
\begin{aligned}
\tilde{X} & \sim X \cos \phi+Y \sin \phi, \\
\tilde{Y} & \sim-X \sin \phi+Y \cos \phi, \\
\tilde{R} & \sim R .
\end{aligned}
$$

Thus the transfer from Cartesian coordinates to the local coordinates is, to leading order, simply a rotation by the angle $\phi$. The vectors $\boldsymbol{b}$ and $\boldsymbol{n}$ are found to be

$$
\boldsymbol{b} \sim\left(\begin{array}{c}
-\sin \phi \\
\cos \phi \\
0
\end{array}\right), \quad \boldsymbol{n} \sim\left(\begin{array}{c}
\cos \phi \\
\sin \phi \\
0
\end{array}\right),
$$

and the $\tilde{\boldsymbol{e}}_{\theta}(s)$ unit vector is given by

$$
\tilde{\boldsymbol{e}}_{\theta} \sim \boldsymbol{e}_{\theta}+O(\epsilon)
$$

In terms of the Cartesian coordinates, equation (4.11) states that

$$
\begin{aligned}
\boldsymbol{J} \sim & \frac{\boldsymbol{e}_{\theta}}{R}\left\{\kappa+\frac{K(X \cos \phi+Y \sin \phi)}{2}\right\} \\
& +\frac{K}{2}\left(-\log \left(\frac{R}{\kappa}\right)+\log 2-1\right)\left(\begin{array}{c}
-\sin \phi \\
\cos \phi
\end{array}\right)+\cdots,
\end{aligned}
$$

which gives

$$
\boldsymbol{J} \sim \frac{\kappa}{R} \boldsymbol{e}_{\theta}+\frac{K \log \kappa}{2 \sqrt{g_{x 0}^{2}+g_{y 0}^{2}}}\left(\begin{array}{c}
g_{y 0} \\
-g_{x 0}
\end{array}\right)
$$



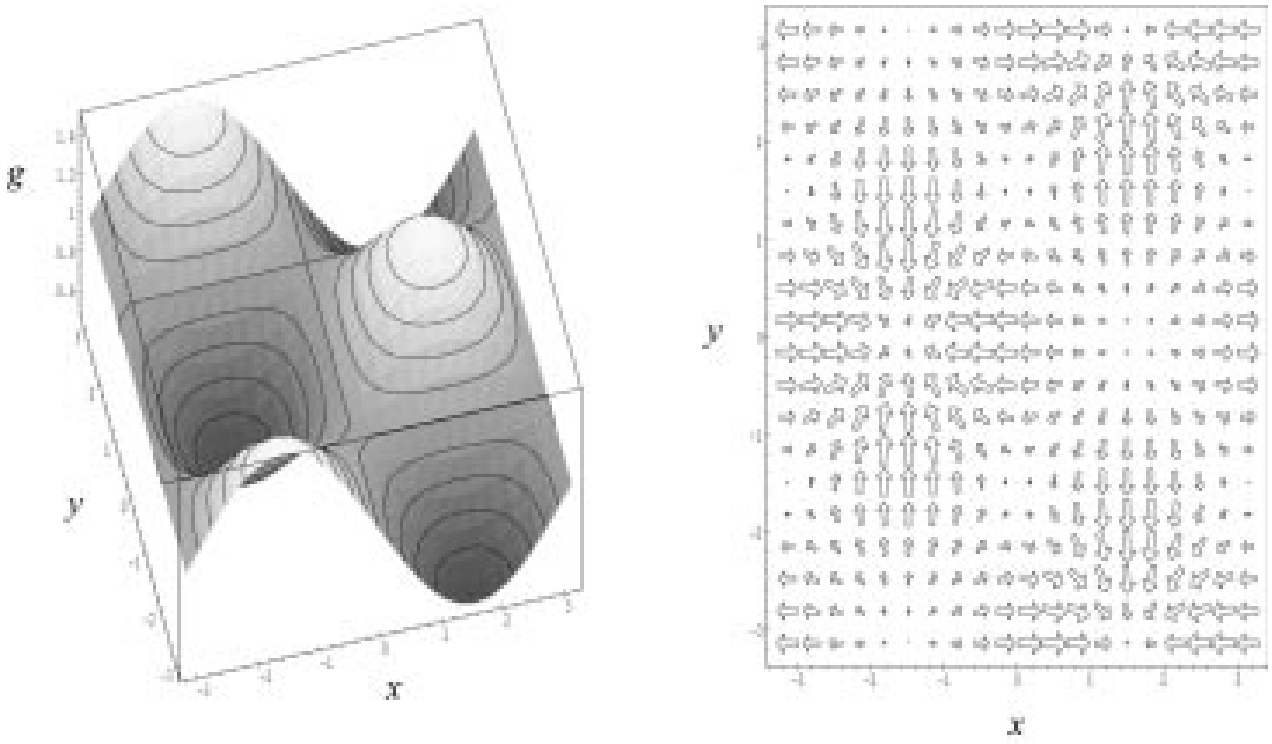

FIG. 4.2. A sample film of varying thickness and the velocity field it generates.

since, for $\Gamma$ to meet normally with the surfaces of the film,

$$
\cos \phi \sim-\frac{g_{x 0}}{\sqrt{g_{x 0}^{2}+g_{y 0}^{2}}}, \quad \sin \phi \sim-\frac{g_{y 0}}{\sqrt{g_{x 0}^{2}+g_{y 0}^{2}}} .
$$

Thus equation (4.19) matches equation (4.9) with $\Gamma$ having constant curvature given by

$$
K \sim \frac{\sqrt{g_{x 0}^{2}+g_{y 0}^{2}}}{g_{0}},
$$

that is, $\Gamma$ is an arc of a circle which meets the surfaces of the film normally at $\left(0,0, \pm \epsilon g_{0}\right)$. The matching with the vortex core then follows as in [3], and the velocity of the vortex is given by (4.12) to be

$$
\boldsymbol{v} \sim-\left.\frac{\log \kappa}{\beta} \nabla(\log g)\right|_{(0,0)} .
$$

The velocity is therefore in the direction of the $x, y$ components of the normal to the surface. This implies that, in the absence of any external forces, a vortex will move toward the locally thinnest part of the film, where it will be straight and therefore stationary. We show $g$ and $-\nabla(\log g)$ for the case

$$
g=1+\frac{1}{2} \sin x \cos y,
$$

\section{in Figure 4.2.}

Remark. We see that the velocity generated due to variations in film thickness is $O(\log \kappa)$. If we proceeded to the next term in $\boldsymbol{v}$ we would find, as in [3], that the velocity generated by neighboring vortices or the applied field is $O(1)$. Thus the motion 
will be dominated by (4.22) for low applied magnetic fields and for well-separated vortices. When that applied magnetic field becomes $O(\log \kappa)$, or the separation of vortices becomes $O\left((\log \kappa)^{-1 / 2}\right)$, their effect on vortex motion will be in balance with the effects of varying thickness. For stronger applied magnetic fields, or for vortices closer together, the effects of varying thickness will become negligible. However, since the vortex separation tends to zero as $\kappa \rightarrow \infty$ in these last two cases, the "outer" model on the $\lambda$ lengthscale will involve a homogenized vortex density, or vorticity, in place of the sum of $\delta$-functions. We will return to such homogenized models at the end of the next section.

5. Modified models for films of differing lateral extent. Let us now consider the effect of a more general $l \gg \epsilon$, with $a \sim \delta \sim l$ as before. We rescale onto this $l$ lengthscale using $\boldsymbol{r}=l \hat{\boldsymbol{r}}$. The equations (3.1) to (3.8) then become

$$
\begin{aligned}
\frac{1}{l} \nabla_{\wedge} \boldsymbol{J}+\boldsymbol{H} & =\frac{2 \pi}{l^{2}} \sum_{i} \delta\left(\hat{\boldsymbol{x}}-\hat{\boldsymbol{x}}_{i}\right) \boldsymbol{e}_{z}, \quad \hat{x}, \hat{y} \in \hat{\Omega} \text { and } \quad|\hat{z}|<\frac{\epsilon}{l} g, \\
\boldsymbol{J} & =\frac{1}{l} \boldsymbol{\nabla}_{\wedge} \boldsymbol{H}, \quad \hat{x}, \hat{y} \in \hat{\Omega} \text { and } \quad|\hat{z}|<\frac{\epsilon}{l} g, \\
\boldsymbol{\nabla} \cdot \boldsymbol{H} & =0, \quad \hat{x}, \hat{y} \in \hat{\Omega} \text { and } \quad|\hat{z}|<\frac{\epsilon}{l} g, \\
\boldsymbol{\nabla}_{\wedge} \boldsymbol{h} & =\mathbf{0}, \quad \hat{x}, \hat{y} \notin \hat{\Omega} \text { or } \quad|\hat{z}|>\frac{\epsilon}{l} g \\
\boldsymbol{\nabla} \cdot \boldsymbol{h} & =0, \quad \hat{x}, \hat{y} \notin \hat{\Omega} \text { or } \quad|\hat{z}|>\frac{\epsilon}{l} g
\end{aligned}
$$

with

$$
\begin{aligned}
\boldsymbol{h}=\boldsymbol{H} & \text { on } \hat{x}, \hat{y} \in \hat{\Omega} \text { and }|\hat{z}|=\frac{\epsilon}{l} g, \\
\boldsymbol{J} \cdot \boldsymbol{\nu}=0 & \text { on } \partial \hat{\Omega} \text { and }|\hat{z}|<\frac{\epsilon}{l} g,
\end{aligned}
$$

and

$$
\boldsymbol{h} \rightarrow h_{A} \boldsymbol{e}_{z} \quad \text { as } \quad|\hat{\boldsymbol{r}}| \rightarrow \infty .
$$

Although it may appear that $l \ll 1$ or $l \gg 1$ will decide the behavior of these equations we will see below that the product $\epsilon l$ is the important parameter. We recall that an $O(|\boldsymbol{J}|)$ current in a film of thickness order $\epsilon$ produces an $O(\epsilon|\boldsymbol{J}|)$ external magnetic field. Noting that the vortices are separated by order-one distances on this new lengthscale, the right-hand side of equation (5.1) implies we should rescale

$$
\begin{aligned}
\boldsymbol{h} & =\frac{1}{l^{2}} \tilde{\boldsymbol{h}}, \\
\boldsymbol{H} & =\frac{1}{l^{2}} \tilde{\boldsymbol{H}}, \\
\boldsymbol{J} & =\frac{1}{\epsilon l^{2}} \tilde{\boldsymbol{J}} .
\end{aligned}
$$

We found in the previous section that the current density generated in a sample of lateral size $l$ by an applied field $h_{A}$ was $O\left(l h_{A}\right)$, while that generated by the vortices 
was $O(1 / l)$. To keep these two currents in balance we will assume that the applied field is $O\left(1 / l^{2}\right)$, so that we will also rescale

$$
h_{A}=\frac{1}{l^{2}} \tilde{h}_{A} .
$$

If we rescale $\hat{z}=\frac{\epsilon}{l} Z$ to look inside the film, equations (5.1) to (5.3) become

$$
\begin{aligned}
-\frac{1}{\epsilon^{2}} \frac{\partial \tilde{J}_{2}}{\partial Z}+\frac{1}{\epsilon l} \frac{\partial \tilde{J}_{3}}{\partial \hat{y}}+\tilde{H}_{1} & =0, \\
\frac{1}{\epsilon^{2}} \frac{\partial \tilde{J}_{1}}{\partial Z}-\frac{1}{\epsilon l} \frac{\partial \tilde{J}_{3}}{\partial \hat{x}}+\tilde{H}_{2} & =0, \\
\frac{1}{\epsilon l}\left(\frac{\partial \tilde{J}_{2}}{\partial \hat{x}}-\frac{\partial \tilde{J}_{1}}{\partial \hat{y}}\right)+\tilde{H}_{3} & =2 \pi \sum_{i} \delta\left(\hat{\boldsymbol{x}}-\hat{\boldsymbol{x}}_{i}\right), \\
\tilde{J}_{1} & =-\frac{\partial \tilde{H}_{2}}{\partial Z}+\frac{\epsilon}{l} \frac{\partial \tilde{H}_{3}}{\partial \hat{y}}, \\
\tilde{J}_{2} & =\frac{\partial \tilde{H}_{1}}{\partial Z}-\frac{\epsilon}{l} \frac{\partial \tilde{H}_{3}}{\partial \hat{x}}, \\
\tilde{J}_{3} & =\frac{\epsilon}{l}\left(\frac{\partial \tilde{H}_{2}}{\partial \hat{x}}-\frac{\partial \tilde{H}_{1}}{\partial \hat{y}}\right), \\
\frac{\partial \tilde{H}_{1}}{\partial \hat{x}}+\frac{\partial \tilde{H}_{2}}{\partial \hat{y}}+\frac{l}{\epsilon} \frac{\partial \tilde{H}_{3}}{\partial Z} & =0 .
\end{aligned}
$$

We see that equation (5.15) has a distinguished limit when $\Lambda=1 / \epsilon l \sim 1$. As we shall see, the size of this parameter $\Lambda$ has a dramatic effect on the model. For the case $\Lambda \sim 1$ (or $l \sim 1 / \epsilon$ ) we can find the leading-order solution exactly as in section 3.1, which is

$$
\begin{aligned}
\Lambda \boldsymbol{e}_{z} \cdot \nabla_{\wedge} \tilde{\boldsymbol{J}}^{(0)}+\left.\tilde{h}_{3}^{(0)}\right|_{\hat{z}=0^{+}} & =2 \pi \sum_{i} \delta\left(\hat{\boldsymbol{x}}-\hat{\boldsymbol{x}}_{i}\right), \\
\nabla \cdot\left(g \tilde{\boldsymbol{J}}^{(0)}\right) & =0 \\
\boldsymbol{\nabla}_{\wedge} \tilde{\boldsymbol{h}}^{(0)} & =\mathbf{0} \\
\boldsymbol{\nabla} \cdot \tilde{\boldsymbol{h}}^{(0)} & =0
\end{aligned}
$$

with

$$
\begin{aligned}
{\left[\hat{\boldsymbol{e}}_{z} \wedge \hat{\boldsymbol{h}}^{(0)}\right] } & =2 g \tilde{\boldsymbol{J}}^{(0)} \quad \text { for } \quad \hat{x}, \hat{y} \in \hat{\Omega}, \\
\tilde{\boldsymbol{h}}^{(0)} & \rightarrow \tilde{h}_{A}^{(0)} \hat{\boldsymbol{e}}_{z} \quad \text { as } \quad|\hat{\boldsymbol{r}}| \rightarrow \infty \\
\tilde{\boldsymbol{J}}^{(0)} \cdot \hat{\boldsymbol{\nu}} & =0 \quad \text { on } \quad \partial \hat{\Omega},
\end{aligned}
$$

where

$$
\begin{aligned}
\tilde{\boldsymbol{h}} & =\tilde{\boldsymbol{h}}^{(0)}+\cdots, \\
\tilde{h}_{A} & =\tilde{h}_{A}^{(0)}+\cdots, \\
\tilde{\boldsymbol{J}} & =\tilde{\boldsymbol{J}}^{(0)}+\cdots,
\end{aligned}
$$


If $\Lambda$ becomes much greater than 1, it can be seen that the first term in (5.20) becomes dominant, the problem for the leading-order current decouples, and we recover a similar set of equations to (3.39) to (3.42) and (3.44) to (3.46) in section 3.1 (which are for the specific case of $l \sim 1$ or $\Lambda \sim 1 / \epsilon$ ).

With $\Lambda \sim 1$ both terms on the left-hand side of equation (5.20) remain important and the current and external magnetic field are coupled.

When $\Lambda \ll 1$ the leading-order problem becomes

$$
\left.h_{3}^{(1)}\right|_{z=0^{+}}=2 \pi \sum_{i} \delta\left(\boldsymbol{x}-\boldsymbol{x}_{i}\right)
$$

along with equations (5.21) to (5.26). Thus $\left.h_{3}^{(1)}\right|_{z=0^{+}}=0$ away from vortex cores, and an inner analysis is necessary near each vortex in which the neglected derivatives of the current become important. However, this final case becomes much more interesting when the separation of vortices is much less than $l$ and we can homogenize the righthand side into a vorticity, which will be the subject of another paper.

The law of motion (4.22), being derived from a local analysis, is still valid in the present model.

6. Conclusions. We have examined the interaction of superconducting vortices with boundaries in a half-space and a thin film. We found that, as for fluid vortices, superconducting vortices must meet a boundary normally.

We formulated a thin-film model for isolated vortices in section 3, in which the equations for the current in the film and the external magnetic field decouple. We used this model to examine the effect of varying film thickness on the motion of a vortex. We found that vortices would move with a velocity proportional to $-\nabla \log g$, where $g$ is the function describing the film thickness. Such a law of motion is consistent with the vortex moving under the curvature induced by being forced to meet the boundaries of the film normally, and results in the vortex moving to the locally thinnest part of the film.

Finally, we considered the effect of the film having large lateral dimension by comparison with the penetration depth. We formulated a new thin-film model, in which the external magnetic field and the current in the film are again coupled.

For each of the films we have considered, we assumed that the separation of vortices, $a \lambda$, and the lengthscale for the variation in film thickness, $\delta \lambda$, were both of the same order as the lateral dimensions of the film, $l \lambda$, as $\epsilon \rightarrow 0, \kappa \rightarrow \infty$. This means that there is a finite number of vortices in the limit. In this case, since the current generated by a vortex is order one, while the pinning force generated by a nonuniform film is order $\log \kappa$, the pinning forces will dominate the intervortex forces as $\kappa \rightarrow \infty$.

For more general $a$ and $\delta$, the current in the film will be of order $1 / a^{2}$, giving an intervortex force of order $1 / a^{2}$, while the pinning forces will be of order $(1 / \delta) \log \kappa$. Thus the pinning forces will be effective until the current in the film becomes of order $(1 / \delta) \log \kappa$. Since the current generated in the film by an applied field of magnitude $h_{A}$ is of order $l h_{A}$, this corresponds to an applied field of order $1 /(l \delta) \log \kappa$.

Let us now translate this back into dimensional variables. Let $\tilde{l}=l \lambda$ be the dimensional width of the film, and $\tilde{\delta}=\delta \lambda$ be the dimensional lengthscale for variations in film thickness. As a dimensional reference for the magnetic field we can either choose the flux quantum $\Phi_{0}$, which is one in these units, the thermodynamic critical magnetic field $H_{c}$, which is $\kappa / \sqrt{2}$ in these units, or the upper critical field $H_{c_{2}}$, which 
is $\kappa^{2}$ in these units. The pinning force is then effective up to a magnetic field of order

$$
\frac{\lambda^{2}}{\tilde{l} \tilde{\delta}} \log \kappa \Phi_{0}=\frac{\lambda^{2}}{\tilde{l} \tilde{\delta}} \log \kappa \sqrt{2} \frac{H_{c}}{\kappa}=\frac{\lambda^{2}}{\tilde{l} \tilde{\delta}} \log \kappa \frac{H_{c_{2}}}{\kappa^{2}} .
$$

\section{REFERENCES}

[1] E. Brandt And M. Indenbom, Type-II-superconductor strip with current in a perpendicular field, Phys. Rev. B, 48 (1993), p. 12, 893.

[2] S. J. Chapman, Q. Du, And M. D. Gunzburger, A model for variable thickness superconducting thin films, Z. Angew. Math. Phys., 47 (1996), p. 410.

[3] S. J. Chapman and G. Richardson, Motion of vortices in type II superconductors, SIAM J. Appl. Math., 55 (1995), p. 1275.

[4] A. T. Dorsey, Vortex motion and the Hall effect in type-II superconductors: A time-dependent Ginzburg-Landau theory approach, Phys. Rev. B, 46 (1992), p. 8376.

[5] Q. Du And M. D. GunzBurger, A model for superconducting thin films having variable thickness, Phys. D, 69 (1993), p. 215.

[6] W. E, Dynamics of vortices in Ginzburg-Landau theories with applications to superconductivity, Phys. D, 77 (1994), p. 383.

[7] V. L. Ginzburg and L. D. Landau, On the theory of superconductivity, J. Experiment. Theoret. Phys., 20 (1950), p. 1064. [Translation: L. D. Landau, Collected papers of L. D. Landau, Pergamon Press, Oxford, 1965.]

[8] L. P. Gor'Kov and G. M. ÉliashberG, Generalization of the Ginzburg-Landau equations for non-stationary problems in the case of alloys with paramagnetic impurities, Soviet Phys. J.E.T.P., 27 (1968), p. 328.

[9] V. Kogan, Pearl's vortex near the film edge, Phys. Rev. B, 49 (1994), p. 15, 874.

[10] V. Kogan, A. Yu. Simonov, And M. LedviJ, Magnetic field of vortices crossing a superconductor surface, Phys. Rev. B, 48 (1993), p. 392.

[11] M. C. MarchetTi, Modification of the magnetic flux-line interaction at a superconductor's surface, Phys. C 200 (1992), p. 155.

[12] J. C. Neu, Vortices in complex scalar fields, Phys. D, 43 (1990), p. 385.

[13] J. Pearl, Current distribution in superconducting films carrying quantized fluxoids, Appl. Phys. Lett., 5 (1964), p. 65.

[14] J. PeARL, Structure of superconductive vortices near a metal-air interface, J. Appl. Phys., 37 (1966), p. 4139.

[15] L. Peres And J. Rubinstein, Vortex dynamics in U(1) Ginzburg-Landau models, Phys. D, 64 (1993), pp. 299-309.

[16] P. G. Saffman, Vortex Dynamics, Cambridge University Press, Cambridge, UK, 1992.

[17] T. Schuster, H. Kuhn, E. Brandt, M. Indenbom, M. Koblischka, and M. Konczykowski, Flux motion in thin superconductors with inhomogeneous pinning, Phys. Rev. B, 50 (1994), p. 16,684 .

[18] G. Stejic, A. Gurevich, E. Kadyrov, D. Christen, R. Joynt, and D. Larbalestier, Effects of geometry on the critical currents of thin films, Phys. Rev. B, 49 (1994), 1274.

[19] E. Zeldov, J. R. Clem, M. McElfresh, And M. Darwin, Magnetization and transport currents in thin superconducting films, Phys. Rev. B, 49 (1994), p. 9802. 